\title{
BAYESIAN ESTIMATION OF DISTRIBUTED PHENOMENA USING DISCRETIZED REPRESENTATIONS OF PARTIAL DIFFERENTIAL EQUATIONS
}

\author{
Felix Sawo, Kathrin Roberts and Uwe D. Hanebeck \\ Intelligent Sensor-Actuator-Systems Laboratory \\ Institute of Computer Science and Engineering \\ Universität Karlsruhe (TH) \\ Karlsruhe, Germany \\ Email: \{sawo,roberts\}@ira.uka.de,uwe.hanebeck@ieee.org
}

\begin{abstract}
Keywords: Stochastic systems, Baysian estimation, model-based reconstruction, distributed phenomenon, environmental monitoring, sensor-actuator-network.

Abstract: $\quad$ This paper addresses a systematic method for the reconstruction and the prediction of a distributed phenomenon characterized by partial differential equations, which is monitored by a sensor network. In the first step, the infinite-dimensional partial differential equation, i.e. distributed-parameter system, is spatially and temporally decomposed leading to a finite-dimensional state space form. In the next step, the state of the resulting lumped-parameter system, which provides an approximation of the solution of the underlying partial differential equations, is dynamically estimated under consideration of uncertainties both occurring in the system and arising from noisy measurements. By using the estimation results, several additional tasks can be achieved by the sensor network, e.g. optimal sensor placement, optimal scheduling, and model improvement. The performance of the proposed model-based reconstruction method is demonstrated by means of simulations.
\end{abstract}

\section{INTRODUCTION}

Recent developments in wireless sensor network technology and miniaturization of sensor nodes make it possible to exploit centralized (Kumar et al., 2002) and decentralized (Rao et al., 1993) sensor networks for monitoring the natural environment (Thuraisingham, 2004; Culler and Mulder, 2004). Such sensor networks can be used for example in industrial, medical, urban, and many other environments. Furthermore, applying sensor networks can provide new data for environmental science, such as climate models, as well as growth and reproduction of coral reef indicated by physical environmental factors, e.g. temperature, water movement, salt concentration, and pollutant concentration. Further examples are reconstruction of fluid-flow in a thermal reactor (Faulds and King, 2000), and reconstruction of the surface motion of a beating heart in minimally invasive surgery (Ortmaier et al., 2005).

In practical implementations the sensor nodes are normally densely deployed either inside the phenomenon or very close to it. In order to get meaningful information not only at the sensor nodes itself but also between these nodes, the reconstruction of the distributed phenomenon is of major significance.
For the prediction of the state of such phenomenon we present a new method for systematic data processing. Basically, data from the sensor network in form of measurement results as well as data from a physical model are used to derive the best possible estimate for the state of a distributed phenomenon; even at nonmeasurement points. The estimation results on the other hand can be used for finding the optimal placement and measurement time sequences for the sensor nodes, or for improving the parameter values of the used physical model, see Fig. 1.

However, for a model-based approach it is necessary to solve the partial differential equations characterizing the distributed phenomena. Such equations can be solved for example by the finite-element method or the finite-difference method (Baker, 1983). The unstructured nature of those methods permits a good geometrical description of complex geometries. Additionally, it is possible to solve even nonlinear partial differential equations. However, many parameters describing uniquely the state of the distributed phenomenon are necessary to achieve appropriate convergence.

On the other hand, there are modal analysis methods using a set of global expansion functions. These methods just need a few parameters for characterizing 


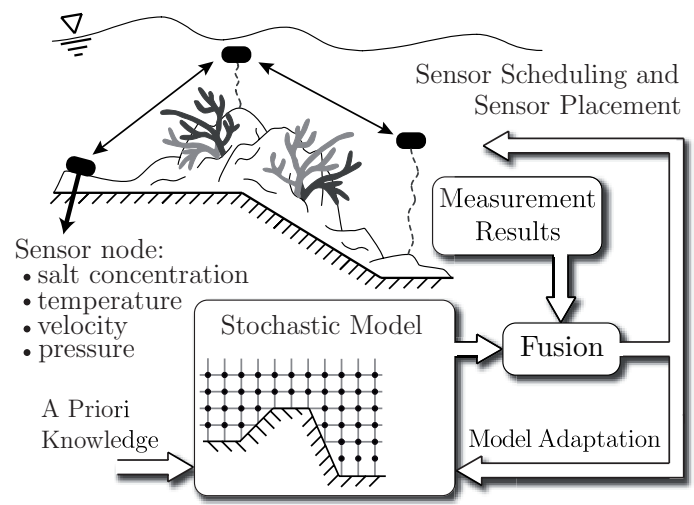

Figure 1: Visionary scenario for the reconstruction and the observation of a distributed phenomenon by means of a sensor network; observation of a coral reef.

a smooth solution of the partial differential equation (Roberts and Hanebeck, 2005). The drawback, however, is that the modal analysis works only for linear partial differential equations and global expansion functions can be found only for problems with simple boundary conditions.

Combining these two general approaches, leads to the so-called finite-spectral method (Karniadakis and Sherwin, 2005; Fournier et al., 2004; Levin et al., 2000). Basically, this method approximates the solution within each element with a set of orthogonal polynomials, such as Legendre, or Chebyshev. Thus, it combines the geometrical flexibility of conventional finite-element methods with the exponential convergence rate associated with the modal analysis.

The novelty of this paper is the model-based observation of distributed phenomena by a sensor network under consideration of uncertainties both occuring in the physical model, i.e., system model, and arising from noisy measurements. Here, we extend and generalize our previous research work (Roberts and Hanebeck, 2005) in such a way that both the system model and the measurement model are derived by the finite-spectral method. Using this method, it turns out that nonlinear phenomena with complex boundary conditions can be reconstructed and predicted in a systematic manner.

The remainder of this paper is organized as follows. In Section II, a rigorous formulation of the problem of the reconstruction of distributed phenomena characterized by partial differential equations is given. In Section III and IV, the spatial and temporal decomposition of the partial differential equation is shown. Finally, in Section V the centralized estimator is derived and its performance is demonstrated by means of some simulation results; reconstruction of the temperature distribution in a heat rod by means of a sensor network.

\section{PROBLEM FORMULATION}

The main goal is to design a dynamic system model for the purpose of estimating the state of a distributed phenomenon monitored by a sensor network. A large number of distributed phenomena, such as irrotational fluid flow, heat conduction, and wave propagation (Roberts and Hanebeck, 2005), can be described by means of a set of linear partial differential equations.

In this paper, a one-dimensional partial differential equation is used for simplicity, given by

$$
\frac{\partial f(z, t)}{\partial t}-c \frac{\partial^{2} f(z, t)}{\partial z^{2}}-s(z, t)=\mathbb{L}(f)=0,
$$

where $f(z, t)$ denotes the solution of the partial differential equation, e.g. temperature at a certain location $z$ and at a certain time $t, s(z, t)$ represents the source term, and $c$ is a positive constant. Considering the solution in a domain $\Omega=\{z \mid 0 \leq z \leq L\}$, we assume following boundary conditions

$$
f(z=L, t)=g_{D}, \quad \frac{\partial f(z=0, t)}{\partial z}=g_{N},
$$

where $g_{D}$ is referred to as a Dirichlet boundary condition and $g_{N}$, specifying a condition on the derivative, is the so-called Neumann boundary condition.

The above mentioned partial differential equation (1) describes the distributed phenomenon in an infinite-dimensional state space. However, in order to estimate and reconstruct the state of a distributed phenomenon by means of a Bayesian estimation approach, the partial differential equation has to be characterized by a finite state space form according to

$$
\underline{x}_{k+1}=\mathbf{A}_{k} \underline{x}_{k}+\mathbf{B}_{k} \underline{u}_{k}+\underline{w}_{k},
$$

where $\underline{x}_{k}$ contains the individual states characterizing the time evolution of the distributed phenomenon and the matrix $\mathbf{A}_{k}$ maps the current state vector at time step $k$ to the next state vector at time step $k+1$. The noise vector $\underline{w}_{k}$ contains both driving input $\Delta \underline{u}_{k}$ noises and subsumed endogenous uncertainties $\underline{d}_{k}$, e.g. modeling errors, according to

$$
\underline{w}_{k}=\left[\begin{array}{ll}
\mathbf{I} & \mathbf{I}
\end{array}\right]\left[\begin{array}{c}
\mathbf{B} \Delta \underline{u}_{k} \\
\underline{d}_{k}
\end{array}\right],
$$

where $\mathbf{I}$ is the identity matrix.

Furthermore, it is assumed that the measurements $\underline{\hat{y}}_{k}$ are related linearly to the state vector $\underline{x}_{k}$, according $\frac{y}{\text { to }}$

$$
\underline{\hat{y}}_{k}=\mathbf{H}_{k} \underline{x}_{k}+\underline{v}_{k},
$$

where $\mathbf{H}_{k}$ denotes the measurement gain matrix and $\underline{v}_{k}$ represents the measurement uncertainties.

Once the system equation and the measurement equation is derived, the state vector $\underline{x}_{k}$ characterizing the distributed phenomena can be estimated by means of the Kalman filter for the linear case (Anderson and Moore, 1979), or nonlinear estimation procedures for the nonlinear case, e.g. (Hanebeck et al., 2003). 


\section{SPATIAL DECOMPOSITION (ODE-SYSTEM)}

In this section we present the spatial decomposition allowing the conversion of the partial differential equation (1) into a set of ordinary differential equations (ODE-System). It is well-known that the finite-difference, the finite-element, and the finitespectral method may be used with the same numerical methodologies described in the next section, namely the Galerkin formulation (Baker, 1983; Karniadakis and Sherwin, 2005).

\subsection{Galerkin Formulation}

The first basic ingredient of a spatial decomposition method is an approximate solution replacing the infinite expansion by a finite representation. Thus, it is assumed that the solution $f(z, t)$ can be represented by a piecewise approximation $\tilde{f}(z, t)$ according to

$$
\tilde{f}(z, t)=\sum_{i=0}^{N_{d o f}-1} \Psi_{i}(z) \alpha_{i}(t)
$$

where $\Psi_{i}(z)$ are analytic functions called the shape functions, e.g. see Fig. 2 (b), and $N_{d o f}$ denotes the degree of freedom of the resulting system description.

The essence of all spatial decomposition methods such as the finite-difference, the finite-element, and the finite-spectral method lies in the choice of the shape functions $\Psi_{i}(z)$. This function is to be specified in more detail later.

Due to the fact that such approximated solution cannot satisfy the partial differential equation (1) everywhere in the region of interest a residual is left. A common method known as the Galerkin formulation is based on finding a way to make this residual small in some sense; achieved by minimizing an appropriate number of weighted integrals. The most popular choice for the weighting functions is the shape function itself. Replacing both the solution function $f(z, t)$ and the input function $s(z, t)$ by a finite expansion (4), denoted by $\tilde{f}(z, t)$ and $\tilde{s}(z, t)$, respectively, leads to following weighted integral over the solution domain $\Omega$

$$
\int_{\Omega} \Psi_{i}(z)\left[\frac{\partial \tilde{f}(z, t)}{\partial t}-c \frac{\partial^{2} \tilde{f}(z, t)}{\partial z^{2}}-\tilde{s}(z, t)\right] d z=0
$$

It can be stated that the minimization of this integral automatically leads to the best numerical approximation of the solution $f(z, t)$ of the partial differential equation (Baker, 1983).

Then, the integral of (5) can be recasted by using the rules of product differentiation. Applying the fundamental theorem of calculus for the evaluation of the
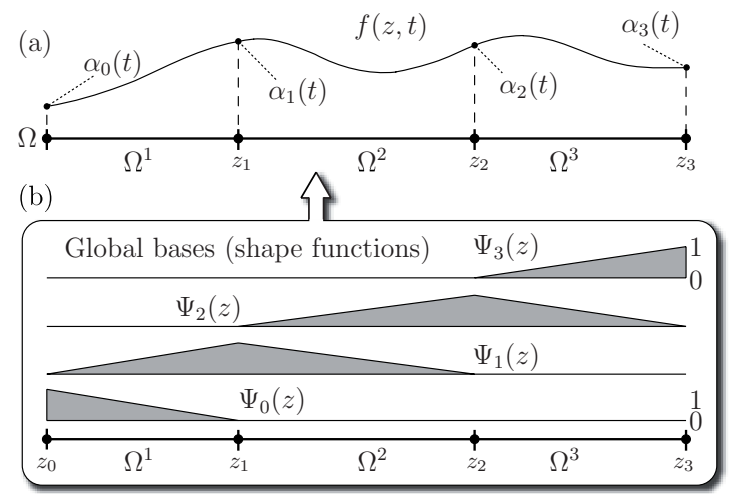

Figure 2: The solution $f(z, t)$ of the partial differential equation is approximated by $\tilde{f}(z, t)$ depending on the shape functions $\Psi_{j}(z)$ and their weighting coefficients $\alpha_{j}(\mathrm{t})$. (a) Elemental decomposition of the solution domain $\Omega$ into several subdomains $\Omega^{e}$. (b) Shape functions $\Psi_{i}(z)$ for the linear case.

resulting integral leads to

$$
\begin{aligned}
& \int_{\Omega} \Psi_{i}(z) \frac{\partial \tilde{f}(z, t)}{\partial t} d z=\int_{\Omega} \Psi_{i}(z) \tilde{s}(z, t) d z \\
& -c \int_{\Omega} \frac{d \Psi_{i}(z)}{d z} \frac{\partial \tilde{f}(z, t)}{\partial z} d z+c\left[\Psi_{i}(z) \frac{\partial \tilde{f}(z, t)}{\partial z}\right]_{\partial \Omega_{l}}^{\partial \Omega_{r}}
\end{aligned}
$$

where the last term on the right-hand side contains the boundary conditions, i.e., $\partial \Omega_{r}$ and $\partial \Omega_{l}$ denotes the right-hand and left-hand boundary, respectively. This weighted residual statement is known as the weak formulation of the partial differential equation (1).

As it is shown in the next sections, by specifying the shape functions $\Psi_{i}(z)$ the weighted residual statement (6) can be reduced to a system of ordinary differential equations in $\alpha_{i}(t)$, i.e., the semi-discrete version of problem (1),

$$
\mathbf{M}_{G} \underline{\dot{x}}(t)=\mathbf{M}_{G} \underline{u}(t)-c \mathbf{D}_{G} \underline{x}(t)+\underline{b}^{*}(t) .
$$

In this system, $\mathbf{M}_{G}$ is called the global mass matrix and $\mathbf{D}_{G}$ is the global diffusion matrix, and $\underline{b}^{*}$ represents the boundary conditions. The vector $\underline{x}(t)$ and $\underline{\dot{x}}(t)$ are the state vectors of the unknown weighting coefficients $\alpha_{i}(z)$ and their derivatives

$$
\underline{x}(t)=\left[\alpha_{0}(t), \alpha_{1}(t), \cdots, \alpha_{N_{d o f}-1}(t)\right]^{T} .
$$

It is important to note that the entries of the matrices $\mathbf{M}_{G}$ and $\mathbf{D}_{G}$, and the state vector $\underline{x}(t)$ merely depend upon the choice of the shape functions $\Psi_{i}(z)$, as shown in the following sections.

The next sections are devoted to appropriate definitions of the shape functions $\Psi_{i}(z)$. First, the $h$ type expansion functions decomposing the solution domain into small subdomains are considered; $\Psi_{i}(z)$ 
are usually linear functions or simple polynomials. Then, based on this decomposition it is possible to introduce additional supporting nodes within the subdomains; the so-called nodal $p$-type expansion functions using higher-order orthogonal polynomials. Finally, the shape functions $\Psi_{i}(z)$ can be defined by a set of orthogonal polynomials of different order or modes, called modal $p$-type expansion functions. For each type it is shown that the global mass matrix $\mathbf{M}_{G}$ and the global diffusion matrix $\mathbf{D}_{G}$ can be calculated in a similar manner.

\subsection{Elemental Decomposition (h-type extension)}

In this section, the decomposition of the solution domain $\Omega$ into small subdomains $\Omega^{e}$ is demonstrated in more detail, which is referred to as the h-type extension process. This process basically reduces the sizes of the individual subdomains $\Omega^{e}$ in order to achieve convergence of the approximated solution.

Here, the decomposition process is explained and visualized by means of simple polynomials for the shape functions $\Psi_{i}(z)$. However, the same techniques can be exploited with higher-order polynomial expressions, as shown in the next sections. The decomposition of the solution domain into subdomains and the respective shape functions for linear polynomials are visualized in Fig. 2.

Substituting the finite expansion (4) into the weak formulation (6) of the partial differential equation yields the following individual terms of the weak formulation

$$
\begin{aligned}
\int_{\Omega} \Psi_{i}(z) \frac{\partial \tilde{f}(z, t)}{\partial t} d z & =\sum_{j=0}^{N_{d o f}-1} M_{i j}^{g} \frac{d \alpha_{j}(t)}{d t} \\
\int_{\Omega} \frac{d \Psi_{i}(z)}{d z} \frac{\partial \tilde{f}(z, t)}{\partial z} d z & =\sum_{j=0}^{N_{d o f}-1} D_{i j}^{g} \alpha_{j}(t) \\
\int_{\Omega} \Psi_{i}(z) \tilde{s}(z, t) d z & =\sum_{j=0}^{N_{d o f}-1} M_{i j}^{g} \hat{s}_{j}(t)
\end{aligned}
$$

The individual entries $M_{i j}^{g}$ and $D_{i j}^{g}$, defined by

$$
\begin{aligned}
M_{i j}^{g} & =\int_{\Omega} \Psi_{i}(z) \Psi_{j}(z) d z, \\
D_{i j}^{g} & =\int_{\Omega} \frac{d \Psi_{i}(z)}{d z} \frac{d \Psi_{j}(z)}{d z} d z,
\end{aligned}
$$

can be assembled to the global mass matrix $\mathbf{M}_{G}$ and the global diffusion matrix $\mathbf{D}_{G}$, respectively. At this point it can be easily recognized that this always leads to the ordinary differential equation given in (7).

Furthermore, it is noted that in the case of linear shape functions $\Psi_{i}(z)$ this leads to finite-difference
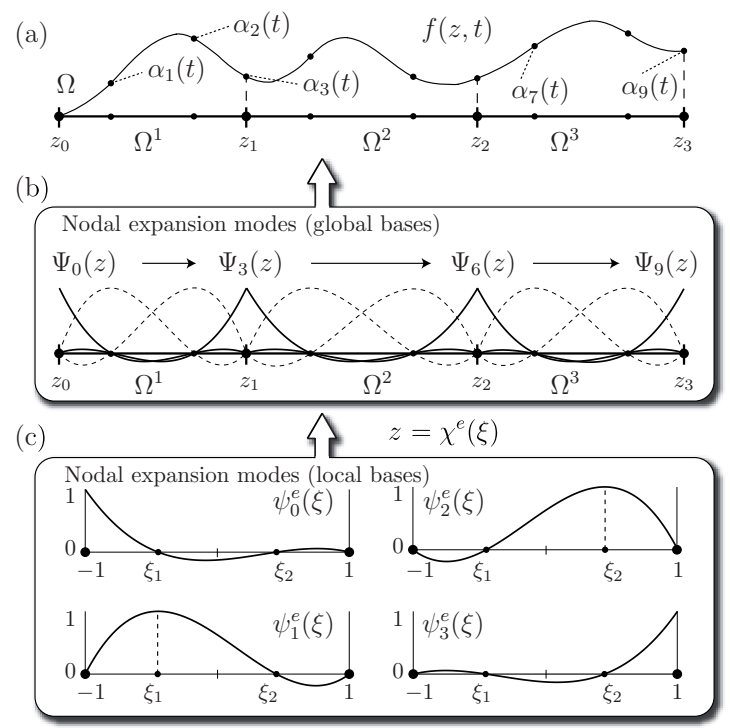

Figure 3: Approximation of the solution $f(z, t)$ by means of a nodal polynomial expansion, e.g. Legendre polynomials for $m=4$ refinement. (a) Elemental decomposition of the solution domain $\Omega$ into several subdomains $\Omega^{e}$. (b) Shape functions $\Psi_{i}(z)$ for a nodal polynomial expansion visualized in global bases, and (c) in local bases (standard element).

formula, which can be regarded as a special case of the finite-element method (Baker, 1983).

\subsection{Nodal Polynomial Expansion}

This section describes how the weak formulation (6) is decomposed in space using higher order polynomials; also called p-type expansion. Assuming a fixed mesh, these methods achieve a higher accuracy of the solution by increasing the polynomial order inside the element.

Since a consideration of the expansion in terms of global modes $\Psi_{i}(z)$ is uneconomical and numerically intractable, especially when using a large number of elements, it is reasonable to introduce a standard element $\Omega_{s t}$, such that

$$
\Omega_{s t}=\{\xi \mid-1 \leq \xi \leq 1\} .
$$

This standard element $\Omega_{s t}$ can be mapped to any elemental domain $\Omega^{e}$ using the isoparametric transformation $\chi^{e}(\xi)$, which expresses the global coordinate $z$ in terms of the local coordinate $\xi$, depicted in Fig. 3 (b)-(c).

A class of nodal $p$-type expansions which also have become known as spectral elements are based on the Legendre polynomials. Those polynomials are de- 
fined in the standard domain $\Omega_{s t}$ according to

$$
L_{m}(\xi)=\frac{1}{2^{m} m !} \frac{d^{m}\left(\xi^{2}-1\right)^{m}}{d \xi^{m}},
$$

where $m$ denotes the degree of the used polynomial. Based on the Legendre polynomials the spectral elements are given by

$$
\psi_{p}^{e}(\xi)=\frac{\left(1-\xi^{2}\right) L_{m}^{\prime}(\xi)}{m(m+1) L_{m}\left(\xi_{p}\right)\left(\xi_{p}-\xi\right)},
$$

where $L_{m}$ is the Legendre polynomial of degree $m$, $L_{m}^{\prime}$ denotes the differentiation with respect to the argument, and $\xi_{p}$ is the $p$-th Gauss-Lobatto-Legendre quadrature point defined by the corresponding root of $\left(1-\xi^{2}\right) L_{m}^{\prime}(\xi)=0$.

The choice of these quadrature point plays an important role in the stability of the approximation. The spectral elements $\psi_{p}^{e}(\xi)$ are shown in the standard domain $\Omega^{e}$ in Fig. (3) for $m=4$, (Fournier et al., 2004).

Finally, considering the approximated solution in terms of the spectral elements $\psi_{p}^{e}(\xi)$, we can express the approximate solution $\tilde{f}(z, t)$ in terms of $\psi_{p}^{e}(\xi)$ according to

$$
\tilde{f}(z, t)=\sum_{j=0}^{N_{d o f}-1} \Psi_{j}(z) \alpha_{j}(t)=\sum_{e=1}^{N_{e l}} \sum_{p=0}^{m} \psi_{p}^{e}(\xi) \alpha_{p}^{e}(t)
$$

where $\psi_{p}^{e}(\xi)=\psi_{p}\left(\left[\chi^{e}\right]^{-1}(z)\right)$ contains the transformation from local bases to global bases in terms of the parametric mapping $\chi^{e}(\xi)$. The coefficients $\alpha_{j}(t)$ in this form have a physical interpretation in that they represent the solution of the partial differential equation (1) at the nodal points $x_{j}$. This fact is exploited for the derivation of a simple measurement gain matrix $\mathbf{H}_{k}$, i.e., it turns out that it is reasonable to put the sensor nodes onto the polynomial nodes, see Sec. 4.2.

Upon substituting the nodal expansion (8) into the weak formulation of the partial differential equation (6) the entries of the local mass matrices $M_{i j}^{e}$ and local diffusion matrices $D_{i j}^{e}$ can be derived as

$$
\begin{aligned}
& M_{i j}^{e}=\int_{-1}^{1} \psi_{i}^{e}(\xi) \psi_{j}^{e}(\xi) d \xi \\
& D_{i j}^{e}=\int_{-1}^{1} \frac{d \psi_{i}^{e}(\xi)}{d \xi} \frac{d \psi_{j}^{e}(\xi)}{d \xi} d \xi .
\end{aligned}
$$

Considering the boundary conditions at the elemental nodes, e.g. $z_{1}$ and $z_{2}$ in Fig. 3, the local matrices $M_{i j}^{e}$ and $D_{i j}^{e}$ can be easily assembled to the global mass matrix $\mathbf{M}_{G}$ and global diffusion matrix $\mathbf{D}_{G}$. This is an automatic procedure known as global assembly.

\subsection{Modal Polynomial Expansions}

For the construction of $p$-type expansions it is often favorable to select a set of orthogonal functions (poly-

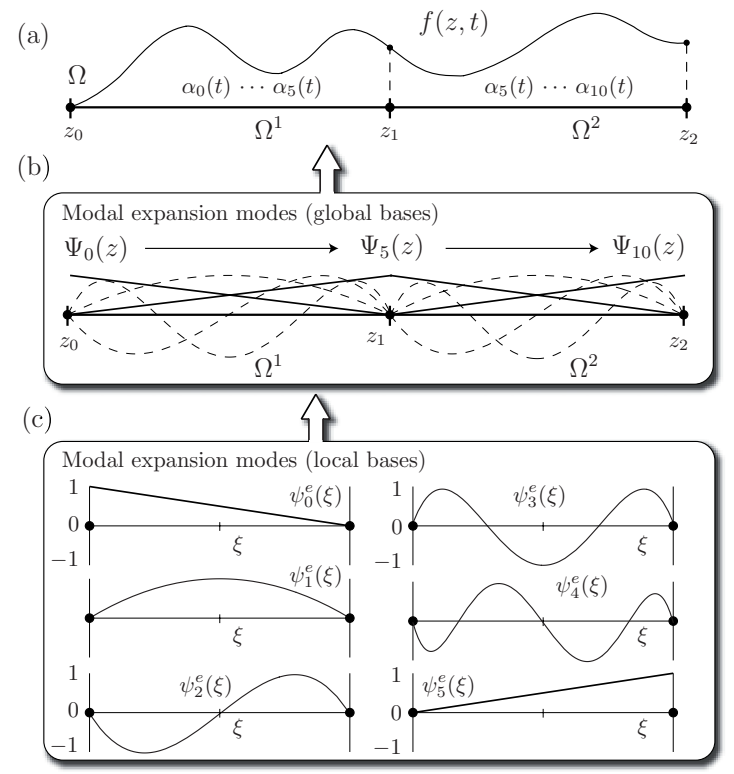

Figure 4: Approximation of the solution $f(z, t)$ by means of a modal polynomial expansion, e.g. Legendre polynomials for $m=5$ refinement. (a) Elemental decomposition of the solution domain $\Omega$ into several subdomains $\Omega^{e}$. (b) Shape functions $\Psi_{i}(z)$ for a modal polynomial expansion visualized in global bases, and (c) in local bases (standard element).

nomials), such as Legendre, Chebyshev or sine functions. The most commonly used orthogonal polynomials in computational fluid dynamics, which offer some advantages compare to the other polynomials, are based upon the Legendre polynomials. This $p$ type modal expansion modes in the standard element $\Omega_{s t}$ are defined as

$$
\psi_{p}^{e}(\xi)=\left\{\begin{array}{cc}
\frac{1-\xi}{2} & p=0 \\
\left(1-\xi^{2}\right) L_{p-1}^{\prime}(\xi) & 0<p<m \\
\frac{1-\xi}{2} & p=m
\end{array},\right.
$$

where the lowest expansion modes $\psi_{0}(\xi)$ and $\psi_{p}(\xi)$ are the same as the linear finite element expansion. These modes are denoted as boundary modes since they are the only modes which are nonzero at the ends of the interval. In Fig. 4 the $p$-type modal expansion based upon the Legendre polynomials is depicted for $m=5$, (Karniadakis and Sherwin, 2005).

Thus, the approximate solution $\tilde{f}(z, t)$ in terms of a modal polynomial expansion can be expressed in the same manner as the nodal polynomial expansion (8); the function $\psi_{p}^{e}(\xi)$ is just replaced by $p$ type modal expansion modes (10) and the coefficients $\alpha_{j}(t)$ denote the weighting coefficients for the individiual modes.

The local mass matrices $M_{i j}^{e}$ and local diffusion 
matrices $D_{i j}^{e}$ can be derived similar to the nodal polynomial expansion (9). Considering the boundary conditions at the end of each element, e.g. $z_{1}$ in Fig. 4, the local matrices $M_{i j}^{e}$ and $D_{i j}^{e}$ can be easily assembled to the global mass matrix $\mathbf{M}_{G}$ and global diffusion matrix $\mathbf{D}_{G}$.

\section{TEMPORAL DISCRETIZATION OF ODE-SYSTEM}

In the previous section we presented the spatial decomposition which allows the conversion of the partial differential equation into a set of ordinary differential equation. In this section, we are now ready to specifiy the time evolution leading to the discrete-time system equation (2) and the discrete-time measurement equation (3).

\subsection{System Equation}

To circumvent the restriction on the time step, $\Delta t$, it is reasonable to integrate the set of ordinary differential equations (7) by means of implicit methods, such as the Crank-Nicolson discretization. This produces a linear system of equations for the state vector $\underline{x}_{k+1}$ containing the unknown weighting factors $\alpha_{i}$ at the $k+1$ time step, which is unconditionally stable. Adding noise terms and modelling error terms leads to following system equation

$$
\underline{x}_{k+1}=\mathbf{A}_{k} \underline{x}_{k}+\mathbf{B}_{k} \underline{u}_{k}+\underline{b}_{k}+\underline{w}_{k},
$$

where the matrices $\mathbf{A}_{k}$ and $\mathbf{B}_{k}$ are determined using the global mass matrix $\mathbf{M}_{G}$ and the global diffusion matrix $\mathbf{D}_{G}$. The vector $\underline{b}_{k}$ containing the boundary conditions is determined using the matrices $\mathbf{M}_{G}, \mathbf{D}_{G}$, and the boundary vector $\underline{b}_{k}^{*}$

\subsection{Measurement Equation}

This section is devoted to the derivation of the discrete-time measurement equation. The sensor nodes, which are densely deployed inside the distributed phenomenon, are exploited to observe that phenomenon and to improve its estimated state. That means, the measurement of the $i$-th sensor node at the location $z=p_{i}$ and at time step $k$, i.e., $t=t_{k}$, is related to the state vector $\underline{x}_{k}$ according to

$$
\begin{aligned}
\hat{y}_{i}\left(p_{i}, t=t_{k}\right) & =\sum_{j=0}^{N_{d o f}-1} \Psi_{j}\left(p_{i}\right) \alpha_{j}\left(t=t_{k}\right) \\
& =\underline{\Psi}^{T}\left(p_{i}\right) \underline{x}_{k} .
\end{aligned}
$$

Assuming a sensor network consisting of $L$ sensor nodes, the measurement gain matrix $\mathbf{H}_{k}$ is set up by the shape function $\underline{\Psi}^{T}\left(p_{i}\right)$ of order $N$, according to

$$
\underline{\hat{y}}_{k}=\underbrace{\left(\begin{array}{ccc}
\Psi_{1}\left(p_{1}\right) & \cdots & \Psi_{N}\left(p_{1}\right) \\
\vdots & \ddots & \vdots \\
\Psi_{1}\left(p_{L}\right) & \cdots & \Psi_{N}\left(p_{L}\right)
\end{array}\right)}_{\mathbf{H}_{k}} \underline{x}_{k}+\underline{v}_{k}
$$

where $\underline{v}_{k}$ are the measurement uncertainties. The number of rows $L$ of the matrix $\mathbf{H}_{k}$ depends on the number of used sensors, i.e., number of measurement points, and the number of columns $N$ depends on the desired number of modes.

However, in the case of nodal expansion and assuming that the sensors are located at the polynomial nodes the weighting coefficients can be measured directly, i.e., $\alpha_{j}(t)=\hat{y}_{j}$. In other words, the diagonal entries of the measurement gain matrix $\mathbf{H}_{k}$ are either 1 or 0 , depending on the location of the sensor nodes,

$$
\underline{\hat{y}}_{k}=\underbrace{\left(\begin{array}{ccc}
1 & \cdots & 0 \\
\vdots & \ddots & \vdots \\
0 & \cdots & 1
\end{array}\right)}_{\mathbf{H}_{k}} \underline{x}_{k}+\underline{v}_{k} .
$$

\section{CENTRALIZED ESTIMATION APPROACH}

In this section the prediction step and the filter step for a centralized estimation approach are derived. Due to the fact that both the system equation (11) and the measurement equation (3) are linear, it is sufficient to use the linear Kalman filter to obtain the best possible estimate for the system state characterizing the distributed phenomena.

\subsection{Prediction Step}

The purpose of the prediction step is to propagate the current state estimate $\underline{\hat{x}}_{k}^{e}$ through the system equation (11) to the next time step. Thus, the mean of the predicted state vector $\underline{\hat{x}}_{k+1}^{p}$ at time step $k+1$ is given by

$$
\underline{\hat{x}}_{k+1}^{p}=\mathbf{A}_{k} \underline{\hat{x}}_{k}^{e}+\mathbf{B}_{k} \underline{\hat{u}}_{k}+\underline{\hat{b}}_{k} .
$$

Assuming that the input vector $\underline{u}_{k}$ and the state vector $\underline{x}_{k}$ are uncorrelated the covariance matrix is obviously given by

$$
\mathbf{C}_{k+1}^{p}=\mathbf{A}_{k} \mathbf{C}_{k}^{e} \mathbf{A}_{k}^{T}+\mathbf{B}_{k} \mathbf{C}_{k}^{u} \mathbf{B}_{k}^{T}+\mathbf{C}_{k}^{d},
$$

where $\mathbf{C}_{k}^{u}$ is the covariance matrix of the input noise, and $\mathbf{C}_{k}^{d}$ is the covariance matrix of subsumed endogenous uncertainties, e.g. modeling errors. 


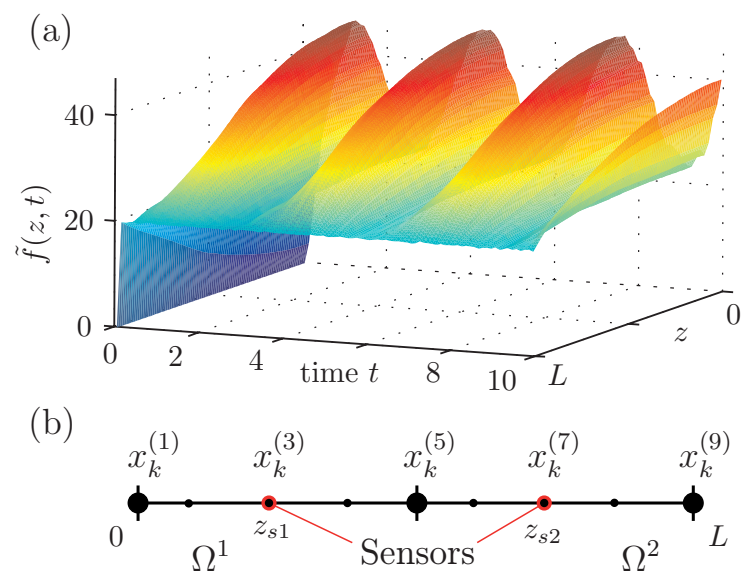

Figure 5: (a) Estimated solution $\tilde{f}(z, t)$. (b) Elemental decomposition into two elements $\Omega_{1}$ and $\Omega_{2}$ with a polynomial expansion of order $m=5$ and location of sensor nodes.

\subsection{Filter Step}

For the purpose of reducing the estimation uncertainty, measurements are used that are related to the state via the measurement equation (3). Given the predicted state $\underline{\hat{x}}_{k}^{p}$ with covariance $\mathbf{C}_{k}^{p}$ and a vector observation $\hat{y}_{k}$ with covariance $\mathbf{C}_{k}^{v}$, the estimated state vector $\underline{\hat{x}}_{k}^{e} \overline{\text { is derived by }}$

$$
\begin{aligned}
\underline{\hat{x}}_{k}^{e}= & \underline{\hat{x}}_{k}^{p}+\mathbf{C}_{k}^{p} \mathbf{H}_{k}^{T}\left(\mathbf{C}_{k}^{v}+\mathbf{H}_{k} \mathbf{C}_{k}^{p} \mathbf{H}_{k}^{T}\right)^{-1} \\
& \cdot\left(\underline{\hat{y}}_{k}-\mathbf{H}_{k} \underline{\hat{x}}_{k}^{p}\right)
\end{aligned}
$$

In the uncorrelated case the covariance matrix of the estimate is given by

$$
\mathbf{C}_{k}^{e}=\mathbf{C}_{k}^{p}-\mathbf{C}_{k}^{p} \mathbf{H}_{k}^{T}\left(\mathbf{C}_{k}^{v}+\mathbf{H}_{k} \mathbf{C}_{k}^{p} \mathbf{H}_{k}^{T}\right)^{-1} \mathbf{H}_{k} \mathbf{C}_{k}^{p}
$$

\section{SIMULATION RESULTS}

In this section we demonstrate the performance of the proposed estimation method by means of some simulation results. The goal is the reconstruction of the temperature distribution in a heat rod using both a physical model and measurements obtained by a sensor network. It is important to note that the novelty of our approach is to consider the uncertainties arising from noisy measurements and occuring in the physical model (state vector and input vector).

The evolution of the temperature is modelled by the well-known one-dimensional heat equation (1) of a bar with the length $L=1 \mathrm{~m}$. The noisy input function is given by $s(z, t)=30 \sin (2 t)+30+\Delta u_{k}$, where $\Delta u_{k}$ denotes the input uncertainties. Applying

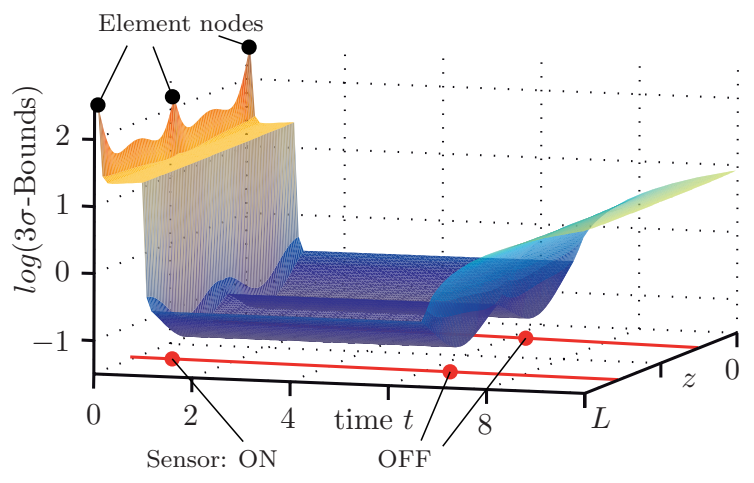

Figure 6: Logarithm of the $3 \sigma$-Bounds as a measure for the uncertainty of the estimated solution $\tilde{f}(z, t)$. Due to the measurements the uncertainty can be decreased rapidly, even between the sensor nodes.

a nodal expansion (8) for the approximated solution $\tilde{f}(z, t)=\underline{\Psi}^{T}(z) \underline{\alpha}(t)$, the infinite-dimensional partial differential equation (1) can be spatially and temporally decomposed leading to the finite-dimensional state space form (11). The state vector $\underline{x}_{k}$ can be derived by temporal discretization of the weighting factors $\underline{\alpha}(t)$ of the approximated solution, as shown in Sec. 4 . Here, we assume a nodal expansion based on a Legendre polynomial of the order $m=5$. The spatial decomposition of the heat rod into two elements $\Omega_{1}$ and $\Omega_{2}$ is visualized in Fig. 5 (b).

Furthermore, it is assumed that for simplicity the sensor network consists of two sensor nodes located at $z_{s 1}=0.25$ and $z_{s 2}=0.75$, shown in Fig. 5 (b). In the case of a nodal expansion the general relation between the measurement vector $\underline{\hat{y}}_{k}$ and the state vector $\underline{x}_{k}$ described by (12) can be simplified to (13). For this simulation example that means the state variables $x_{k}^{(3)}$ and $x_{k}^{(7)}$ can be measured directly, as it is visualized in Fig. 5 (b).

The estimated solution $\tilde{f}(z, t)$ is visualized in Fig. 5 (a) and the logarithm of the $3 \sigma$-bound of the estimated solution $\tilde{f}(z, t)$, which is a measure for its uncertainty, is depicted in Fig. 6. It is obvious that the measurements are used for rapidly decreasing the uncertainty. Due to the model-based approach they can be decreased even between the sensor nodes; the remaining uncertainty merely arises from the model uncertainties.

The estimation results for both a measurement point and a non-measurement point are visualized in Fig. 7. Again, it can be easily seen that the solution $\tilde{f}(z, t)$ is estimated even at non-measurement points with an appropriate certainty. Furthermore, it is clear that by means of the measurements the estimated solution $\tilde{f}(z, t)$ can be significantly improved. 

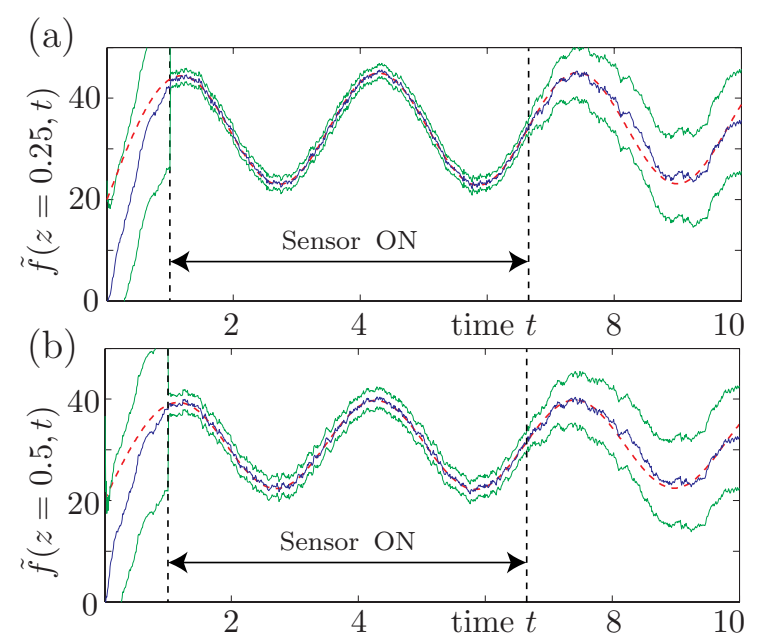

Figure 7: Estimated solution $\tilde{f}(z, t)$ (blue), $3 \sigma$-Bounds (green), and analytic solution $f(z, t)$ (red) at (a) measurement and (b) non-measurement point.

\section{CONCLUSION}

This paper introduced the methodology for deriving system models and measurement models for the reconstruction of distributed phenomena characterized by means of linear partial differential equations. Thanks to the inhomogeneous approximation capabilities and the systematic manner of this approach, the estimation of nonlinear phenomena even with complex geometries is possible. The novelty of this paper is the model-based reconstruction of distributed phenomena under the consideration of uncertainties both occuring in the physical model and arising from noisy measurements.

It is believed that applying such methods in sensor network applications for the reconstruction provides novel prospects to optimal sensor node placement, optimal measurement time sequences, and improvement of the used physical model. The performance of the proposed model-based approach for the reconstruction of distributed pheonmena was demonstrated by means of simulation results for the one-dimensional partial differential equation.

Although for the one-dimensional partial differential equation, the decomposition may seem unnecessarily involved; however, the same principles can easily be applied to the decomposition in multiple dimensions. This is left for future research work. Furthermore, especially for large sensor networks it is essential to find a decentralized reconstruction approach. It is believed that the decomposition method introduced in this paper is well-suited for such an estimation approach.

\section{ACKNOWLEDGEMENTS}

This work was partially supported by the German Research Foundation (DFG) within the Research Training Group GRK 1194 "Self-organizing SensorActuator-Networks".

\section{REFERENCES}

Anderson, B. D. O. and Moore, J. B. (1979). Optimal Filtering. Prentice-Hall.

Baker, A. J. (1983). Finite Element Computational Fluid Mechanics. Taylor and Francis.

Culler, D. E. and Mulder, H. (2004). Smart sensors to network the world. In Scientific American.

Faulds, A. L. and King, B. B. (2000). Sensor location in feedback control of partial differential equation systems. In CCA'O0, Proceedings of the 2000 IEEE International Conference on Control Applications.

Fournier, A., Bunge, H.-P., Hollerbach, R., and Vilotte, J.-P. (2004). Application of the spectral-element method to the axisymmetric navier-stokes equation. In Geophysical Journal International.

Hanebeck, U. D., Briechle, K., and Rauh, A. (2003). Progressive bayes: A new framework for nonlinear state estimation. In Proceedings of SPIE, AeroSense Symposium.

Karniadakis, G. E. and Sherwin, S. (2005). Spectral/hp Element Methods for Computational Fluid Dynamics. Oxford University Press.

Kumar, T., Zhao, F., and Shepherd, D. (2002). Collaborative signal and information processing in microsensor networks. In IEEE Signal Processing Magazine.

Levin, J. G., Iskandarani, M., and Haidvogel, D. B. (2000). A nonconforming spectral element ocean model. In International Journal for Numerical Methods in Fluids.

Ortmaier, T., Groeger, M., Boehm, D. H., Falk, V., and Hirzinger, G. (2005). Motion estimation in beating heart surgery. In IEEE Transactions on Biomedical Engineering.

Rao, B., Durrant-Whyte, H., and Sheen, J. (1993). A fully decentralized multisensor system for tracking and surveillance. In International Journal of Robotics Research.

Roberts, K. and Hanebeck, U. D. (2005). Prediction and reconstruction of distributed dynamic phenomena characterized by linear partial differential equations. In Fusion'05, The Eight International Conference on Information Fusion.

Thuraisingham, B. (2004). Secure sensor information management and mining. In IEEE Signal Processing Magazine. 